\title{
Feasible Modified Subgradient Method for Solving the Thermal Unit Commitment Problem as a New Approach
}

\author{
Ummuhan Basaran Filik and Mehmet Kurban \\ Department of Electrical and Electronics Engineering, Anadolu University, 26555 Eskisehir, Turkey \\ Correspondence should be addressed to Mehmet Kurban, mkurban@anadolu.edu.tr
}

Received 22 December 2009; Accepted 15 June 2010

Academic Editor: Wei-Chiang Hong

Copyright (c) 2010 U. B. Filik and M. Kurban. This is an open access article distributed under the Creative Commons Attribution License, which permits unrestricted use, distribution, and reproduction in any medium, provided the original work is properly cited.

The Lagrangian relaxation- (LR-) based methods are commonly used to solve the thermal unit commitment (UC) problem which is an important subject in power system engineering. The main drawback of this group of methods is the difference between the dual and the primal solutions which gives some significant problems on the quality of the feasible solutions. In this paper, a new approach, feasible modified subgradient (F-MSG) method which does not require finding an unconstrained global minimum of the Lagrangian function and knowing an optimal value of the problem under consideration in order to update dual variables at the each iteration, is firstly used for solving the thermal UC problem. The major advantage of the proposed approach is that it guarantees the zero duality gap and convergence independently from the size of the problem. In order to discuss the advantages of this method, the four-unit Tuncbilek thermal plant, which is located in Kutahya region in Turkey, is chosen as a small test system. The numerical results show that F-MSG gives better solutions as compared to the standard LR method.

\section{Introduction}

Solving thermal UC problem is very important for power system optimization. The thermal UC problem, which is defined as a nonlinear, mixed-integer, and nonconvex, is considered to be an NP (Nonlinear-Programming) hard problem. The high dimension of the possible solution space is the real difficulty in solving the problem. There have been various methods for solving the thermal UC problem in the literature. They are based on mathematical programming and metaheuristic-based approaches. These methods are priority list [1-3], dynamic programming [4], simulated annealing [5-7], tabu search [8, 9], evolutionary programming [10,11], constraint logic programming [12], expert systems [13], fuzzy systems [14], genetic algorithms [15-19], LR [20-24], interior point algorithm [25], memetic algorithm $[26]$, neural network $[27,28]$, and hybrid models $[29,30]$. 
LR which is most widely used approach among the aforementioned methodologies solves the thermal UC problem by "relaxing" or temporarily ignoring the coupling constraints and solving the problem as if they did not exist. Based on the duality theory, the LR method subsequently tries to find the values of the Lagrange multipliers that maximize the dual objective function. The duality gap, a major problem in the nonlinear programming, has been long recognized as an inherent disadvantage of these methods. If the LR-based methods are used for solving the UC problem, dual solution may be far away from the optimal solution. The duality gap for the problem of thermal UC is an important measure of the quality of the solution, which is defined by the smaller gap, the better solution [31]. In [32], different mathematical-based methods LR, penalty function, and augmented Lagrangian penalty function are compared to each other according to feasible cost, dual cost, duality gap, number of iterations, and duration time. According to [32], it is seen that there are differences between primal value and dual value. In the nonlinear programming, the duality gap has been investigated and the theoretical tools for zero duality gap condition have been improved extensively in [33-38].

In this paper, one of the methods based on dual optimization technique, F-MSG method, which has the best performance in eliminating the duality gap in the literature, is firstly used for solving the thermal UC problem as a novel approach. A dual problem with respect to the sharp augmented Lagrangian is constructed for this problem. F-MSG method solves without any duality gap for large class of nonconvex constrained problems. This proposed approach is compared to LR method to show that F-MSG gives an optimal solution obtaining the zero duality gap value. The LR method chosen in testing of F-MSG methods' performance lies from the fact that LR method is the most widely used method in solving UC problems in the literature. In order to compare these methods, the UC problem for fourunit Tuncbilek thermal plant, which is in Kutahya region in Turkey, is solved using MATLAB and General Algebraic Modeling System (GAMS). Note that there is a general acceptance that whenever the system size gets smaller, the duality gap value gets bigger. However, it is shown that this is not the case with the F-MSG method for a small size system [38].

The remaining sections are outlined as follows. Section 2 addresses the thermal UC problem formulations. This section includes an objective function and constraints of the problem. In Section 3, usage of F-MSG method for solving the thermal UC problem is explained in detail. In Section 4, numerical results for F-MSG and LR methods are provided. Finally the conclusions are presented, the main contributions of this paper are highlighted, and possible research directions are discussed.

\section{Review of Thermal Unit Commitment Problem}

The objective of thermal UC problem is to minimize the production cost over the scheduled time horizon (e.g., 8 hours, 24 hours) under the generator operational and spinning reserve constraints while all generating unit constraints are satisfied. The objective function of the UC problem can be formulated as

$$
F\left(P_{i}^{t}, U_{i}^{t}\right)=\sum_{t=1}^{T} \sum_{i=1}^{N}\left[F_{i}\left(P_{i}^{t}\right)+\mathrm{SC}_{i}^{t}\left(1-U_{i}^{t-1}\right)\right] U_{i}^{t}
$$

where $F_{i}\left(P_{i}^{t}\right)=a\left(P_{i}^{t}\right)^{2}+b P_{i}^{t}+c$ is the polynomial function [39]. 
The minimization of the objective function is provided to the following constraints.

(a) Power Balance Constraint. For satisfying the load balance in each stage, the forecasted load demand should be equal to the total power of the generated power for feasible combination:

$$
\sum_{i=1}^{N} U_{i}^{t} P_{i}^{t}=P_{d}^{t}
$$

(b) Spinning Reserve Constraints. In order to meet any abnormal operating conditions, the spinning reserve must be considered:

$$
P_{d}^{t}+P_{r}^{t}-\sum_{i=1}^{N} P_{i, \max } U_{i}^{t} \leq 0 .
$$

(c) Generation Limits Constraint. Each unit must satisfy the generation range:

$$
P_{i, \min } U_{i}^{t} \leq P_{i}^{t} \leq P_{i, \max } U_{i}^{t}, \quad i=1,2, \ldots, N
$$

(d) Ramp-Up and Ramp-Down Constraints. For each unit, output is limited by ramp up/down rate at each hour as follows:

$$
\begin{aligned}
& P_{i}^{t-1}-P_{i}^{t} \leq \mathrm{RD}_{i}, \quad \text { if } U_{i}^{t}=1, U_{i}^{t-1}=1, \\
& P_{i}^{t}-P_{i}^{t-1} \leq \mathrm{RU}_{i}, \quad \text { if } U_{i}^{t}=1, U_{i}^{t-1}=1 .
\end{aligned}
$$

(e) Minimum Up-Down Time Constraint. Once the unit is started up, it should not be shut down before a minimum up-time period is met as follows, and once the unit is shut down, there is a minimum down time before it can be started up again:

$$
\begin{gathered}
\left(T_{i}^{\mathrm{on}(t-1)}-T_{i, \mathrm{up}}^{t}\right)\left(U_{i}^{t-1}-U_{i}^{t}\right) \geq 0, \\
\left(T_{i}^{\mathrm{off}(t-1)}-T_{i, \text { down }}^{t}\right)\left(U_{i}^{t}-U_{i}^{t-1}\right) \geq 0 .
\end{gathered}
$$

The problem is structured binary variables making it clearly nonconvex. These binary variables cause a great deal of trouble and difficulty in solving the UC. Load balance and spinning reserve constraints are coupling constraints for the thermal UC problem.

\section{F-MSG Method for Thermal Unit Commitment}

The F-MSG method guarantees a zero duality gap for a wide class of nonconvex optimization problems. It does not require finding an unconstrained global minimum of the Lagrangian function and knowing an optimal value of the problem under consideration in order to update dual variables at the each iteration, and it guarantees convergence. 


\subsection{F-MSG Algorithm}

Minimize $f_{0}(x)$ subject to $x \in S$ satisfying $f(x)=0$, where $S$ is a subset of a metric space $X$, and $f_{0}: X \rightarrow R$ and $f: X \rightarrow R^{p}$ are given functions.

Sharp Augmented Lagrangian is defined as follows:

$$
L_{\mathrm{sa}}(x, v, c)=f_{0}(x)+c\|f(x)\|-\langle v, f(x)\rangle
$$

and defining the dual function as

$$
H(v, c)=\min _{x \in \Omega} L(x, v, c) .
$$

The dual problem $\left(P^{*}\right)$ is

$$
\max _{(v, c) \in R^{3} x R_{+}} H(v, c) .
$$

Using the above definitions, the F-MSG is as follows [38].

\section{F-MSG Algorithm}

Step 1. Choose positive numbers $\varepsilon_{1} \varepsilon_{2}, \Delta_{1}, M$ and a number $H_{1}$. Set $n=1, t=0$, and $q=0$.

Step 2. Choose $\left(v_{1}^{n}, c_{1}^{n}\right) R_{p} \times R^{+}$and $0<l(1)<M$ set $k=1, v_{k}=v_{1}^{n}, c_{k}=c_{1}^{n}$.

Step 3. Given $\left(v_{k}, c_{k}\right)$, solve the following constraint satisfaction problem $P\left(H_{n}\right)$ : find an element $x \in S$ such that

$$
f_{0}(x)+c\|f(x)\|-\langle v, f(x)\rangle \leq H_{n}
$$

If a solution to (3.4) does not exist, then go to Step 6; otherwise, if a solution $x_{k}$ exists, then check whether $f\left(x_{k}\right)=0$ or not. If $f\left(x_{k}\right)=0$ (or $\|f(x)\| \leq \varepsilon_{1}$ ), then go to Step 5; otherwise go to Step 4.

Step 4. Update dual variables as

$$
\begin{gathered}
v_{k+1}=v_{k}-\alpha s_{k} f\left(x_{k}\right), \\
c_{k+1}=c_{k}+(1+\alpha) s_{k}\left\|f\left(x_{k}\right)\right\| .
\end{gathered}
$$

where $s_{k}$ is a positive step size parameter defined as

$$
\begin{gathered}
0<s_{k}=\frac{\delta \alpha\left(H_{n}-L\left(x_{k}, v_{k}, c_{k}\right)\right)}{\left[\alpha^{2}+(1+\alpha)^{2}\right]\left\|f\left(x_{k}\right)\right\|^{2}}, \\
0<s_{k}=\frac{\left[\delta \alpha\left(H_{n}-L\left(x_{k}, v_{k}, c_{k}\right)\right)+\left(\bar{c}-c_{k}\right)\left\|f\left(x_{k}\right)\right\|\right]}{\left[\alpha^{2}+(1+\alpha)^{2}\right]\left\|f\left(x_{k}\right)\right\|^{2}}
\end{gathered}
$$


with $\alpha>0$, and $0<\delta<2$. In this study, (3.7) is used for updating. The appropriate values are replaced for updating in this equation. We will also require the step size $s_{k}$ corresponding to the dual variables $\left(v_{k}, c_{k}\right)$ to satisfy the following property:

$$
s_{k}\left\|f\left(x_{k}\right)\right\|+c_{k}-\left\|v_{k l}\right\|>l(k) \text {. }
$$

Set $k=k+1$, update $l(k)$ in such a way that $l(k) \rightarrow+\infty$ as $k \rightarrow+\infty$, and repeat Step 3.

Step 5. Let $x_{k}$ be a solution to (3.4) with $f\left(x_{k}\right)=0$. In this case $L\left(x_{k}, v_{k}, c_{k}\right)=f_{0}\left(x_{k}\right)$. Set $q=$ $q+1$ and check $t$. If $t=0$, then set $\Delta_{n+1}=\Delta_{n}$; otherwise set $\Delta_{n+1}=(1 / 2) \Delta_{n}$. Check $\Delta_{n+1}<\varepsilon_{2}$, and then stop. $f_{0}\left(x_{k}\right)$ is an approximate optimal value, $x_{k}$ is an approximate primal solution, and $\left(v_{k}, c_{k}\right)$ is an approximate dual solution; otherwise set $H_{n+1}=\min \left\{f_{0}\left(x_{k}\right), H_{n}-\Delta_{n+1}\right\}$, $n=n+1$, and go to Step 2 .

Step 6. Set $t=t+1$. If $q=0$, then set $\Delta_{n+1}=\Delta_{n}$; otherwise set $\Delta_{n+1}=(1 / 2) \Delta_{n}$. Set $H_{n+1}=$ $H_{n}+\Delta_{n+1}, n=n+1$, and go to Step 2 .

According to F-MSG method for solving the UC problem, the objective function and constraints are defined as follows.

\section{Object Function}

$$
\min _{N, T} f(N, T)=\sum_{t=1}^{T} \sum_{i=1}^{N}\left[F_{i}\left(P_{i}^{t}\right)+\mathrm{SC}_{i}^{t}\left(1-U_{i}^{t-1}\right)\right] U_{i}^{t}
$$

Slack variables are added to inequality constraints. Equality and inequality constraints can be defined as follows.

Generation Limit Constraints

$$
\begin{aligned}
& h_{1}(N, T)=U_{i}^{t} P_{i, \min }-P_{i}^{t}+h_{i}^{t}=0, \\
& h_{2}(N, T)=P_{i}^{t}-U_{i}^{t} P_{i, \max }+h_{i}^{t}=0 .
\end{aligned}
$$

Load Balance Constraints.

$$
h_{3}(T)=\sum_{i=1}^{N} U_{i}^{t} P_{i}^{t}-P_{d}^{t}=0
$$

Spinning Reserve Constraints

$$
h_{4}(T)=P_{d}^{t}+P_{r}^{t}-\sum_{i=1}^{N} P_{i, \max } U_{i}^{t}+h_{i}^{t}=0 .
$$


Ramp-Up and Ramp-Down Constraints

$$
\begin{gathered}
h_{5}(N, T)=P_{i}^{t-1}-P_{i}^{t}-\mathrm{RD}_{i,}+h_{i}^{t}, \quad \text { if } U_{i}^{t}=1, U_{i}^{t-1}=1, \\
h_{6}(N, T)=P_{i}^{t}-P_{i}^{t-1}-\mathrm{RU}_{i}+h_{i}^{t}, \quad \text { if } U_{i}^{t}=1, U_{i}^{t-1}=1 .
\end{gathered}
$$

Minimum Up and Down Constraints

$$
\begin{gathered}
T_{\text {on }}(N, T)=\left(\left(T_{\text {on }}(N, T-1)+1\right)\right) \times U_{i}^{t}, \\
T_{\text {off }}(N, T)=\left(\left(T_{\text {off }}(N, T-1)+1\right)\right) \times\left(1-U_{i}^{t}\right) .
\end{gathered}
$$

$H$ matrix is $=\left[h_{1}(N, T) h_{2}(N, T) h_{3}(T) h_{4}(T) h_{5}(N, T) h_{6}(N, T)\right]$. According to (3.9), sharp augmented Lagrangian is defined as follows:

$$
\begin{aligned}
& L=\sum_{t=1}^{T} \sum_{i=1}^{N}\left[F_{i}\left(P_{i}^{t}\right)+\mathrm{SC}_{i}^{t}\left(1-U_{i}^{t-1}\right)\right] U_{i}^{t} \\
& +c \cdot\left[\operatorname{sqrt}\left(\begin{array}{c}
\left(U_{i}^{t} P_{i, \min }-P_{i}^{t}+h_{i}^{t}\right)^{2}+\left(P_{i}^{t}-U_{i}^{t} P_{i, \max }+h_{i}^{t}\right)^{2} \\
+\left(\sum_{i=1}^{N} U_{i}^{t} \cdot P_{i}^{t}-P_{d}^{t}\right)^{2}+\left(P_{d}^{t}+P_{r}^{t}-\sum_{i=1}^{N} P_{i, \max } U_{i}^{t}+h_{i}^{t}\right)^{2} \\
+\left(P_{i}^{t-1}-P_{i}^{t}-\mathrm{RD}_{i,}+h_{i}^{t}\right)^{2}+\left(P_{i}^{t}-P_{i}^{t-1}-\mathrm{RU}_{i}+h_{i}^{t}\right)^{2}
\end{array}\right)^{2}\right] \\
& \times\left[\begin{array}{c}
-v_{i}^{t} \cdot\left(U_{i}^{t} P_{i, \min }-P_{i}^{t}+h_{i}^{t}\right)-v_{i}^{t} \cdot\left(P_{i}^{t}-U_{i}^{t} P_{i, \max }+h_{i}^{t}\right) \\
-v_{i}^{t} \cdot\left(\sum_{i=1}^{N} U_{i}^{t} \cdot P_{i}^{t}-P_{d}^{t}\right)-v_{i}^{t} \cdot\left(P_{d}^{t}+P_{r}^{t}-\sum_{i=1}^{N} P_{i, \max } U_{i}^{t}+h_{i}^{t}\right) \\
-v_{i}^{t} \cdot\left(P_{i}^{t-1}-P_{i}^{t}-\mathrm{RD}_{i}+h_{i}^{t}\right)-v_{i}^{t} \cdot\left(P_{i}^{t}-P_{i}^{t-1}-\mathrm{RU}_{i}+h_{i}^{t}\right)
\end{array}\right] .
\end{aligned}
$$

The epsilon value is chosen as $0.95 z_{j}$ in the thermal UC problem solution. After (3.15) is constructed using the objective function and constraints, all the steps of the F-MSG method are applied to the thermal UC problem.

\section{Numerical Results}

F-MSG method is applied to the system to find the feasible results and feasible cost, dual cost, and duality gap values. This proposed approach is compared to LR method to show that FMSG gives an optimal solution obtaining the zero duality gap value. The data used in the analysis is taken from Turkish Electric Power Company and Electricity Generation Company. The system parameters and reserve requirements of four units are given in Table 1.

In this study, the 24-hour day is subdivided into 8 discrete stages. The load demands for the stages are given in Table 2 . The spinning reserve demand is set as $10 \%$ of the system 
Table 1: Unit characteristics for four-unit Tuncbilek thermal plant.

\begin{tabular}{lcccccccccc}
\hline $\begin{array}{l}\text { Unit } \\
\text { No }\end{array}$ & $P_{\min }(\mathrm{MW})$ & $P_{\max }(\mathrm{MW})$ & $\mathrm{SC}(\$)$ & $a(\$ / \mathrm{h})$ & $b(\$ / \mathrm{MWh})$ & $c\left(\$ / \mathrm{MW}^{2} \mathrm{~h}\right)$ & $\mathrm{RU}(\$)$ & $\mathrm{RU}(\$)$ & $T_{\text {up }}(\mathrm{h})$ & $T_{\text {down }}(\mathrm{h})$ \\
\hline 1 & 8 & 32 & 60 & 0.515 & 10.86 & 149.9 & 6 & 6 & 1 & 1 \\
2 & 17 & 65 & 240 & 0.227 & 8.341 & 284.6 & 13 & 13 & 2 & 2 \\
3 & 35 & 150 & 550 & 0.082 & 9.9441 & 495.8 & 30 & 30 & 3 & 3 \\
4 & 30 & 150 & 550 & 0.074 & 12.44 & 388.9 & 30 & 30 & 3 & 3 \\
\hline
\end{tabular}

Table 2: Load data (MW).

\begin{tabular}{lccc}
\hline Stage & Load & Stage & Load \\
\hline 1 & 168 & 5 & 313 \\
2 & 150 & 6 & 347 \\
3 & 260 & 7 & 308 \\
4 & 275 & 8 & 231 \\
\hline
\end{tabular}

Table 3: UC schedule for F-MSG method.

\begin{tabular}{|c|c|}
\hline Stage & Unit Combination \\
\hline 1 & 0011 \\
\hline 2 & 0011 \\
\hline 3 & 0111 \\
\hline 4 & 0111 \\
\hline 5 & 1111 \\
\hline 6 & 1111 \\
\hline 7 & 1111 \\
\hline 8 & 0111 \\
\hline
\end{tabular}

demand for each stage. LR method is coded in MATLAB, and F-MSG method is coded in GAMS which is a high-level modeling system for mathematical programming problems [40]. MINOS is used as a GAMS solver. These computer programs are run on Pentium core$1.6 \mathrm{GHz}$ personal computer.

Optimal solution of the dual model set up by the sharp augmented Lagrange function, which is proposed by Gasimov, is equal to the primal solution of the system [37, 38]. The FMSG method is run until the norm is equal to $7.36 \times 10^{-12}$, epsilon is equal to $1.72 \times 10^{7}$, then all the constraints reduce to zero, and feasible solution is obtained. Primal value is equal to the dual value yielding zero duality gap value. The value for the $c$ parameter is 27182.94 and the UC schedule, feasible cost, dual cost, and duality gap values for the F-MSG method are given in Table 3.

The UC schedule, feasible cost, dual cost, and duality gap values for the LR method are given in Table 4.

Feasible cost, dual cost, and duality gap values are found by F-MSG and LR method. These numerical results are given in Table 5 .

It can be seen from Table 5 that LR has the duality gap value and F-MSG performs much better than LR, since duality gap value is reduced to zero. The quality of the solution is improved when the duality gap is decreased. In this paper zero duality gap is achieved and feasible solution is attained by applying the F-MSG method. 
Table 4: UC schedule for LR method.

\begin{tabular}{|c|c|}
\hline $\begin{array}{l}\text { Stage } \\
\text { Sta }\end{array}$ & Unit Combination \\
\hline 1 & $\begin{array}{lllll}0 & 0 & 1 & 1\end{array}$ \\
\hline 2 & 0011 \\
\hline 3 & 0111 \\
\hline 4 & 1111 \\
\hline 5 & 1111 \\
\hline 6 & 1111 \\
\hline 7 & 1111 \\
\hline 8 & 0111 \\
\hline
\end{tabular}

Table 5: The numerical results for F-MSG and LR methods.

\begin{tabular}{lcc}
\hline Compared Items & F-MSG & LR \\
\hline Feasible cost $(\$)$ & 55597.763 & 56443.182 \\
Dual cost $(\$)$ & 55597.763 & 55827.735 \\
Duality gap (\%) & 0 & 1.01102 \\
\hline
\end{tabular}

\section{Conclusion}

The F-MSG method is firstly applied to solve the thermal UC problem which is an important and hard-solving problem in power system engineering. Feasible cost, dual cost, and duality gap values from F-MSG and LR methods are compared. The most attractive feature of the proposed approach is that the duality gap value of F-MSG method over the scheduled time horizon is zero. Also, the total production cost over the scheduled time horizon of the F-MSG method is less than the ones of the standard LR methods. The results of the F-MSG method for solving the thermal UC problem are very impressive and the quality of feasible solution is significantly improved. This approach can be applied to the thermal UC problem for any size of systems to obtain the feasible solutions.

\section{List of Symbols}

$f(x)$ : Objective function

$\bar{H}$ : Upper bound of the dual function

$h(x)$ : Equality constraint

$g(x)$ : Inequality constraint

$N$ : Number of generating units

$P: \quad$ Primal problem

$P^{*}$ : Dual problem

$P_{d}^{t}$ : Nominal demand at hour $t(\mathrm{MW})$

$P_{i}^{t}$ : $\quad$ Generation output of unit $i$ at hour $t(\mathrm{MW})$

$P_{\max , i}^{t}:$ Maximum available capacity of unit $i$ at hour $t(\mathrm{MW})$

$P_{\min , i}^{t}:$ Minimum available capacity of unit $i$ at hour $t$ (MW)

$P_{r}^{t}: \quad$ System spinning reserve at hour $t(\mathrm{MW})$ 


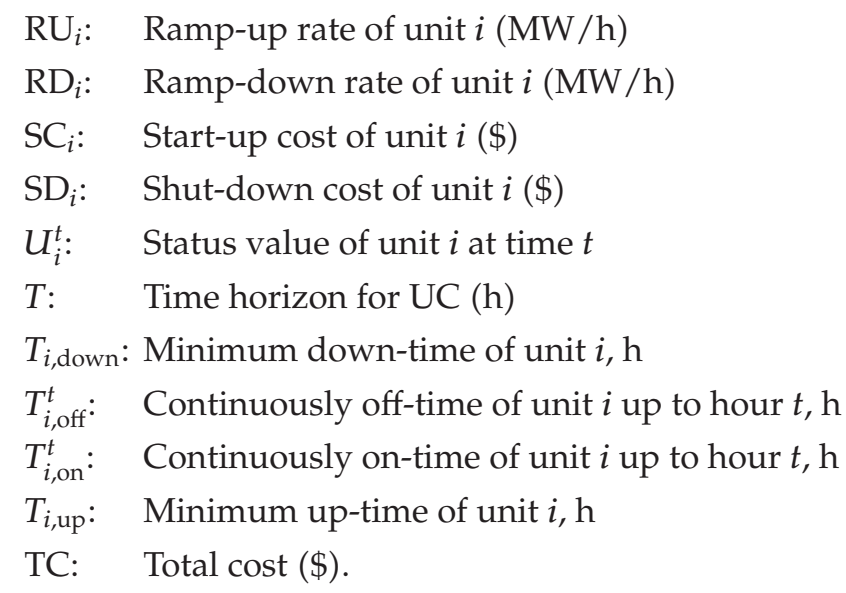

\section{Acknowledgment}

The support and guidance by Professor Rafail N. Gasimov is gratefully acknowledged.

\section{References}

[1] F. N. Lee and Q. Feng, "Multi-area unit commitment," IEEE Transactions on Power Systems, vol. 7, no. 2, pp. 591-599, 1992.

[2] F. N. Lee, "The application of commitment utilization factor (CUF) to thermal unit commitment," IEEE Transactions on Power Systems, vol. 6, no. 2, pp. 691-698, 1991.

[3] J. R. M. Burns and C. A. Gibson, "Optimization of priority lists for a unit commitment program," in Proceedings of IEEE/Power Engineering Society Summer Meeting, vol. 75, p. 453-1, 1975.

[4] C.-L. Chen and N. Chen, "Strategies to improve the dynamic programming for unit commitment application," Transactions of the Chinese Institute of Engineers, vol. 9, no. 3, pp. 181-189, 2002.

[5] F. Zhuang and F. D. Galiana, "Unit commitment by simulated annealing," IEEE Transactions on Power Systems, vol. 5, no. 1, pp. 311-318, 1990.

[6] A. H. Mantawy, Y. L. Abdel-Magid, and S. Z. Selim, "A simulated annealing method for unit commitment," IEEE Transactions on Power Systems, vol. 13, no. 1, pp. 1197-1204, 1998.

[7] A. Viana, J. P. Sousa, and M. Matos, "Simulated annealing for the unit commitment problem," in Proceedings of IEEE Porto Power Tech Conference, Porto, Portugal, September 2001.

[8] H. Mori and T. Usami, "Unit commitment using tabu search with restricted neighborhood," in Proceedings of the International Conference on Intelligent Systems Applications to Power Systems, vol. 221, pp. 422-427, February 1996.

[9] B. Xiaomin, S. M. Shahidehpour, and Y. Erkeng, "Constrained unit commitment by using tabu search algorithm," in Proceedings of the International Conference on Electrical Engineering, vol. 2, pp. 1088-1092, 1996.

[10] C. C. A. Rajan and M. R. Mohan, “An evolutionary programming-based tabu search method for solving the unit commitment problem," IEEE Transactions on Power Systems, vol. 19, no. 1, pp. 577$585,2004$.

[11] K. A. Juste, H. Kita, E. Tanaka, and J. Hasegawa, “An evolutionary programming solution to the unit commitment problem," IEEE Transactions on Power Systems, vol. 14, no. 4, pp. 1452-1459, 1999.

[12] K.-Y. Huang, H.-T. Yang, and C.-L. Huang, "A new thermal unit commitment approach using constraint logic programming," IEEE Transactions on Power Systems, vol. 13, no. 3, pp. 936-945, 1998.

[13] S. Li, S. M. Shahidehpour, and C. Wang, "Promoting the application of expert systems in short-term unit commitment," IEEE Transactions on Power Systems, vol. 8, no. 1, pp. 286-292, 1993.

[14] S. Saneifard, N. R. Prasad, and H. A. Smolleck, "A fuzzy logic approach to unit commitment," IEEE Transactions on Power Systems, vol. 12, no. 2, pp. 988-995, 1997.

[15] K. S. Swarup and S. Yamashiro, "Unit commitment solution methodology using genetic algorithm," IEEE Transactions on Power Systems, vol. 17, no. 1, pp. 87-91, 2002. 
[16] N. P. Padhy, "Unit commitment using hybrid models: a comparative study for dynamic programming, expert system, fuzzy system and genetic algorithms," International Journal of Electrical Power and Energy Systems, vol. 23, no. 8, pp. 827-836, 2001.

[17] T. Senjyu, H. Yamashiro, K. Uezato, and T. Funabashi, "A unit commitment problem by using genetic algorithm based on unit characteristic classification," in Proceedings of IEEE Power Engineering Society Winter Meeting, vol. 1, pp. 58-63, January 2002.

[18] P.-C. Yang, H.-T. Yang, and C.-L. Huang, "Solving the unit commitment problem with a genetic algorithm through a constraint satisfaction technique," Electric Power Systems Research, vol. 37, no. 1, pp. 55-65, 1996.

[19] S. A. Kazarlis, A. G. Bakirtzis, and V. Petridis, "A genetic algorithm solution to the unit commitment problem," IEEE Transactions on Power Systems, vol. 11, no. 1, pp. 83-92, 1996.

[20] N. Jiménez Redondo and A. J. Conejo, "Short-term hydro-thermal coordination by Lagrangian relaxation: solution of the dual problem," IEEE Transactions on Power Systems, vol. 14, no. 1, pp. 89-95, 1999.

[21] Q. Zhai, X. Guan, and J. Cui, “Unit commitment with identical units: successive subproblem solving method based on Lagrangian relaxation," IEEE Transactions on Power Systems, vol. 17, no. 4, pp. 12501257, 2002.

[22] R. Nieva, A. Inda, and I. Guillen, "Lagrangian reduction of search-range for large-scale unit commitment," IEEE Transactions on Power Systems, vol. 2, no. 2, pp. 465-473, 1987.

[23] S. Virmani, E. C. Adrian, K. Imhof, and S. Mukherjee, "Implementation of a Lagrangian relaxation based unit commitment problem," IEEE Transactions on Power Systems, vol. 4, no. 4, pp. 1373-1380, 1989.

[24] W. Ongsakul and N. Petcharaks, "Unit commitment by enhanced adaptive Lagrangian relaxation," IEEE Transactions on Power Systems, vol. 19, no. 1, pp. 620-628, 2004.

[25] L. M. Kimball, K. A. Clements, P. W. Davis, and I. Nejdawi, "Multiperiod hydrothermal economic dispatch by an interior point method," Mathematical Problems in Engineering, vol. 8, no. 1, pp. 33-42, 2002.

[26] J. Valenzuela and A. E. Smith, "A seeded memetic algorithm for large unit commitment problems," Journal of Heuristics, vol. 8, no. 2, pp. 173-195, 2002.

[27] J. Valenzuela and A. E. Smith, "A seeded memetic algorithm for large unit commitment problems," Journal of Heuristics, vol. 8, no. 2, pp. 173-195, 2002.

[28] H. Sasaki, M. Watanabe, J. Kubokawa, N. Yorino, and R. Yokoyama, "A solution method of unit commitment by artificial neural networks," IEEE Transactions on Power Systems, vol. 7, no. 3, pp. 974981, 1992.

[29] N. P. Padhy, "Unit commitment using hybrid models: a comparative study for dynamic programming, expert system, fuzzy system and genetic algorithms," International Journal of Electrical Power and Energy Systems, vol. 23, no. 8, pp. 827-836, 2001.

[30] N. P. Padhy, S. R. Paranjothi, and V. Ramachandran, “A hybrid fuzzy neural network-expert system for a short term unit commitment problem," Microelectronics Reliability, vol. 37, no. 5, pp. 733-737, 1997.

[31] L. A. F. M. Ferreira, "On the duality gap for thermal unit commitment problems," in Proceedings of IEEE International Symposium on Circuits and Systems, vol. 4, pp. 2204-2207, May 1993.

[32] M. Kurban and U. B. Filik, "A comparative study of three different mathematical methods for solving the unit commitment problem," Mathematical Problems in Engineering, vol. 2009, Article ID 368024, 13 pages, 2009.

[33] R. N. Gasimov and A. M. Rubinov, “On augmented lagrangians for optimization problems with a single constraint," Journal of Global Optimization, vol. 28, no. 2, pp. 153-173, 2004.

[34] R. N. Gasimov, "Augmented Lagrangian duality and nondifferantiable optimization methods in nonconvex programming," Journal of Global Optimization, vol. 28, pp. 187-203, 2002.

[35] A. Y. Azimov and R. N. Gasimov, "On weak conjugacy, weak subdifferentials and duality with zero gap in nonconvex optimization," International Journal of Applied Mathematics, vol. 1, pp. 171-192, 1999.

[36] R. T. Rockafellar and R. J. B. Wets, Variational Analysis, Springer, Berlin, Germany, 1998.

[37] A. M. Rubinov and R. N. Gasimov, "Strictly increasing positively homogeneous functions with application to exact penalization," Optimization, vol. 52, no. 1, pp. 1-28, 2003. 
[38] R. N. Gasimov and O. Ustun, "Solving the quadratic assignment problem using F-MSG algorithm," Journal of Industrial and Management Optimization, vol. 3, no. 2, pp. 173-191, 2007.

[39] A. J. Wood and B. F. Wollenberg, Power Generation Operation and Control, John Wiley \& Sons, New York, NY, USA, 2nd edition, 1996.

[40] A. Brooke, D. Kendrick, A. Meeraus, and R. Raman, "GAMS: a users guide," GAMS Development, 1998, http:/ / www.gams.com/. 


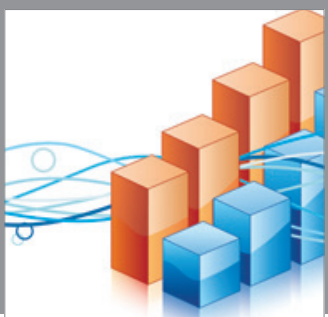

Advances in

Operations Research

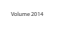

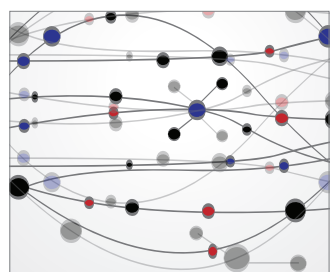

\section{The Scientific} World Journal
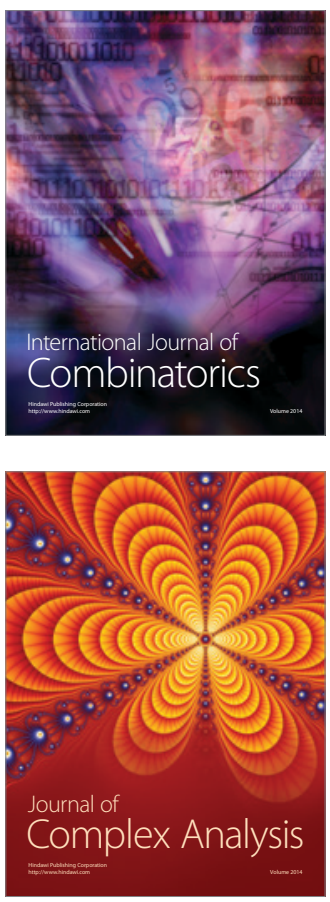

International Journal of

Mathematics and

Mathematical

Sciences
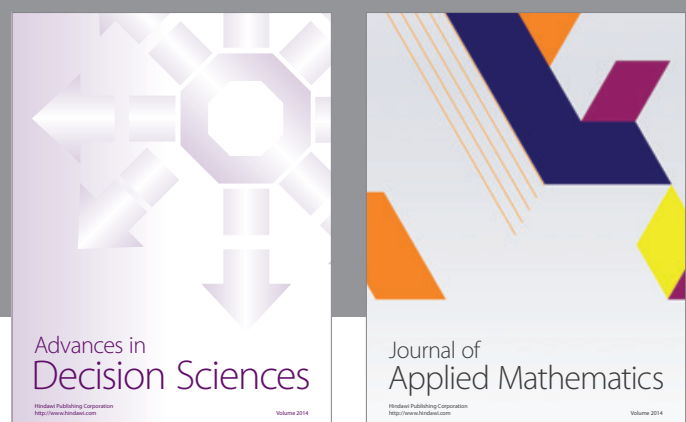

Journal of

Applied Mathematics
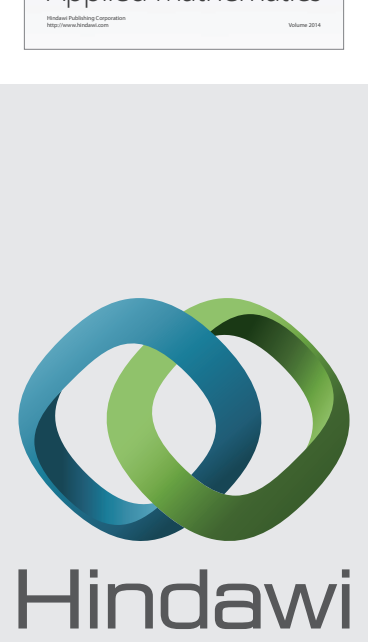

Submit your manuscripts at http://www.hindawi.com
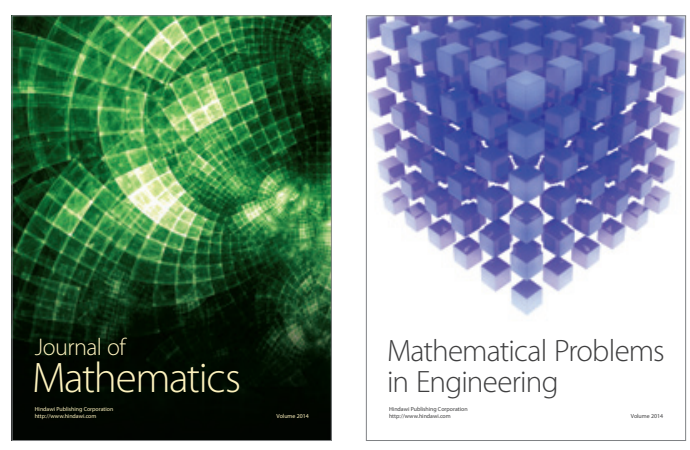

Mathematical Problems in Engineering
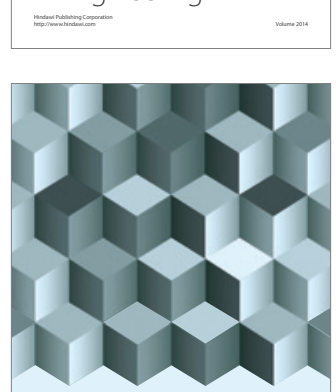

Journal of

Function Spaces
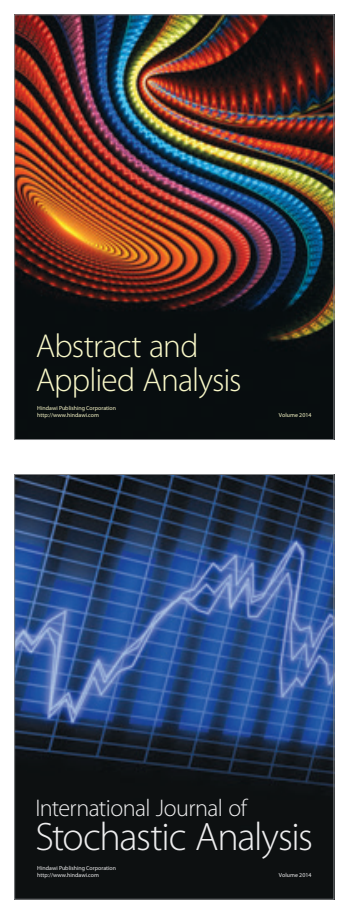

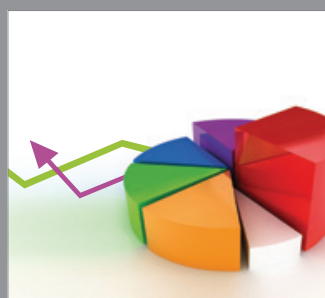

ournal of

Probability and Statistics

Promensencen
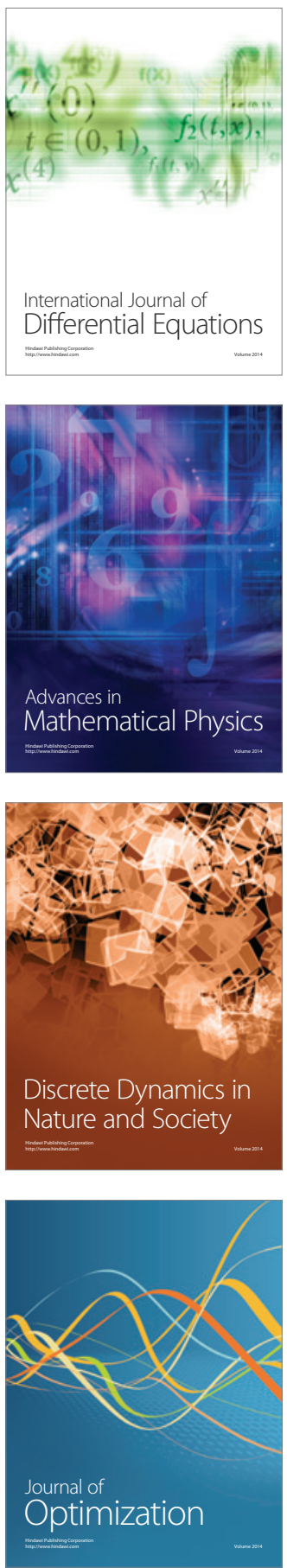